\title{
The Shock Wave Ignition of Dusts
}

\author{
M. Sichel, * S. W. Baek, $\uparrow$ C. W. Kauffman, $\ddagger$ B. Maker $§$ and J. A. Nicholls $\uparrow$ \\ University of Michigan, Ann Arbor, Michigan \\ and \\ P. Wolanski** \\ Technical University of Poland, Warsaw, Poland
}

\begin{abstract}
Dust explosions pose a serious hazard in many industries. The detonability and flammability of dust/oxidizer mixtures depend on the ignition delay of the dust particles when suddenly exposed to a high temperature environment. Consequently, the ignition delay time of dust particles behind a shock wave in the Mach number range of 4.0-5.0 has been measured using a photomultiplier tube to determine the onset of ignition. The dusts investigated included Pittsburgh Seam Coal, graphite, diamond, oats, and RDX. The experimental arrangement, consisting of a shock tube and two different dust injection devices, is described in detail, and experimental results for dusts ranging in particle size from 2 to $74 \mu \mathrm{m}$ are presented. In the Mach number range considered, ignition delay times varied from 2 to $100 \mu \mathrm{s}$. A detailed analytical model based on a solution of the heat conduction equations for the particle interior coupled with a solution of the particle equation of motion has been developed. Heterogeneous reactions occurring on the particle surface and in the pores within the particle are used to model the chemistry. The results were in reasonable agreement with most of the data. Approximate analyses based on a comparison of characteristic thermal and chemical times were also developed. A key conclusion is that the ignition delay is determined mainly by the heat-up time of the particle surface.
\end{abstract}

\section{Nomenclature}

$\begin{array}{ll}A & =\text { pre-exponential factor } \\ B i & =\text { Biot number } \\ C & =\text { specific heat of particle material } \\ C_{D} & =\text { drag coefficient } \\ \bar{C}_{D} & =\text { average drag coefficient } \\ E & =\text { activation energy } \\ h & =\text { film conductance } \\ k_{c} & =\text { thermal conductivity of particle } \\ M & =\text { Mach number } \\ P_{\mathrm{O}_{2}} & =\text { oxygen partial pressure } \\ Q & =\text { combustion heat release per unit mass } \\ R & =\text { particle radius } \\ \bar{R} & =\text { universal gas constant } \\ r & =\text { radius } \\ S_{i} & =\text { internal surface area per unit mass } \\ t & =\text { time } \\ T & =\text { particle temperature } \\ T_{i} & =\text { initial temperature } \\ T_{2} & =\text { static gas temperature behind incident shock } \\ T_{f} & =\text { recovery temperature at particle surface } \\ u^{\prime \prime \prime} & =\text { volumetric heating rate within particle } \\ V_{2} & =\text { gas velocity behind incident shock } \\ V_{p} & =\text { particle velocity } \\ \alpha & =\text { thermal diffusivity of particle } \\ \rho_{2} & =\text { gas density } \\ & \end{array}$

Presented as Paper 84-0205 at the AIAA 22nd Aerospace Sciences Meeting, Reno, Nev., Jan. 9-12, 1984; received July 6, 1984. Copyright (C) American Institute of Aeronautics and Astronautics, Inc., 1984. All rights reserved.

*Professor, Department of Aerospace Engineering. Associate Fellow AIAA.

†Graduate Research Assistant, Department of Aerospace Engineering.

‡Lecturer, Department of Aerospace Engineering. Member AIAA.

§Graduate Research Assistant, Department of Aerospace Engineering. Student Member AIAA.

qProfessor, Department of Aerospace Engineering. Fellow AIAA.

${ }^{* *}$ Associate Professor, Department of Aerospace Engineering.

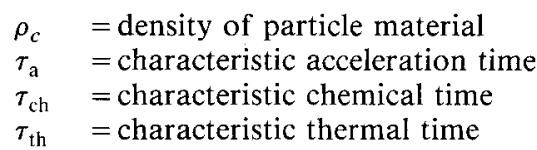

\section{Introduction}

G RAIN dust explosions pose a serious safety hazard for grain elevators, and coal dust explosions can be an important factor in mine accidents. Such explosions may involve accelerating flames or, in some cases, detonations. From a safety point of view, it is important to know the detonability and the detonation and flammability limits of dust clouds, parameters that depend on the detailed structure of the flame or detonation front. The ignition of the dust particles is the first step in dust explosions, and the time required for ignition or the ignition delay has a major influence on flammability and detonability. Anomalies observed in the case of dust explosions, such as the surprising result that RDX clouds of very small particles are more difficult to detonate than clouds with larger particles, ${ }^{1}$ may be related to the details of the processes that lead to particle ignition.

The present paper reports on a combined experimental and analytical study of the shock ignition of coal, graphite, diamond, RDX, and oats dusts and is an extension of the earlier work of Ural et al. ${ }^{2} \mathrm{~A}$ description of the experimental arrangement and the difficulties involved in obtaining reliable data is followed by the presentation of experimental results and a theoretical analysis of the particle ignition process.

\section{Experimental Arrangement}

Experiments were conducted in a horizontal shock tube with a $7.3 \mathrm{~cm}$ diameter driver section $3 \mathrm{~m}$ long and a $3.8 \times 6.4 \mathrm{~cm}$ rectangular driven section $3.6 \mathrm{~m}$ long discharging into a dump tank. The shock tube can produce Mach numbers ranging from 4.0 to 5.0 , which is of the same order as the Mach numbers encountered in detonations.

A small cloud of dust particles is injected into the test section just before the arrival of the shock wave, which is 
monitored through pressure transducers. Light emission from the test section is monitored by a photomultiplier tube, and a neutral density filter is used for decreasing the overall sensitivity. Two pressure switches with a timer are used to measure the shock speed and to trigger an oscilloscope for outputs from the pressure transducer and the photomultiplier tube.

Injection of the dust particles in the shock tube is a major source of difficulty. Two different types of injectors were used. In the first, which is identical to that used by Ural, ${ }^{2}$ dust is loosely packed into grooves at the end of a $6.35 \mathrm{~mm}$ diameter spring driven plunger stopped suddenly at the shock tube surface so that the dust is injected inertially. In the second injector the dust is blown into the test section when a piston is released and expels air from a $25 \mathrm{~mm}$ diameter cylinder, as shown in Fig. 1a. Precise timing is required, to insure that the shock wave strikes the dust just when the injected cloud reaches the center of the test section. It is provided by the injector time delay generator, consisting of a power supply, waveform generator, pulse generator, and oscilloscope. A shadow photograph of the dust cloud is obtained during each test, to monitor whether proper dispersion occurred. A cloud of 53-74 $\mu \mathrm{m}$ graphite dust is shown in Fig. 1c after the passage of a shock with a Mach number of 4.4 , and differential separation of larger and smaller particles is evident.

Extraneous radiation from dirt inside the shock tube was a major problem in initial tests, but was successfully eliminated by passing a strong shock through the tube prior to each test.

The ignition of dust particles in the shock tube was identified by light emission, and the ignition delay time was defined as the time interval between shock-dust contact and the onset of light emission. A precise, absolute determination of the ignition delay is difficult in the experimental arrangement used here. However, it was concluded that consistent use of the same criterion for all experiments would provide good comparative data for different dusts. In the present case, the time at which the deflection of the photomultiplier output trace exceeded the actual trace width was taken as the onset of ignition.

\section{Experimental Results}

Ignition delays in pure oxygen have been measured for the materials listed in Table 1 for values of the post-shock static temperature ranging from 1160 to $1600 \mathrm{~K}$. A summary of the results is presented in Fig. 2, which shows the variation of the ignition delay time on a logarithmic scale with the inverse temperature. This form of representation is typically used for systems governed by an Arrhenius rate law, and then the slope of the delay curve is proportional to the activation temperature. In the present case the ignition delay depends on both the chemistry of ignition and on the physical process of particle heating. Nevertheless, the data still appear to lie on a straight line for the range of experiments. The temperature range above corresponds to Mach numbers ranging from 4 to 5 , as indicated on the Mach number scale above the inverse temperature scale.

Data using both the inertial and the air injector are presented in Fig. 2a. The inertial injector produces clouds with considerable particle agglomeration; consequently, the observed ignition delays are somewhat longer than with the air injector. Straight lines obtained from the data using a mean square fit are shown in Fig. 3. Equivalent activation energies corresponding to the slopes of these curves are also indicated.

Although all the delay times are below $100 \mu \mathrm{s}$, there is a wide discrepancy among the different materials. Oats dust has a significantly shorter delay time than coal, suggesting that it is also a more detonable material. Interestingly, RDX-A (mean diameter $37 \mu \mathrm{m}$ ) with cabosil has shorter

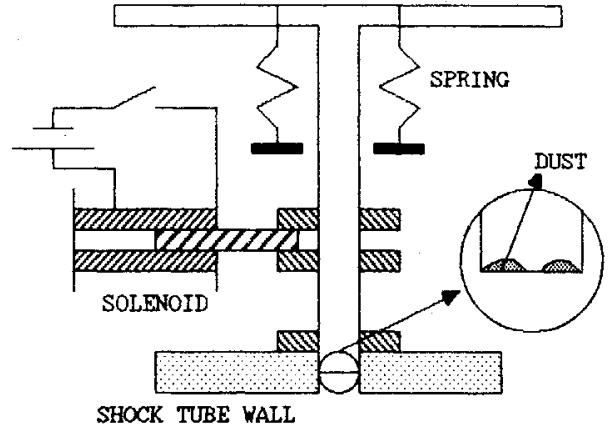

Fig. 1a Inertial dust injector.

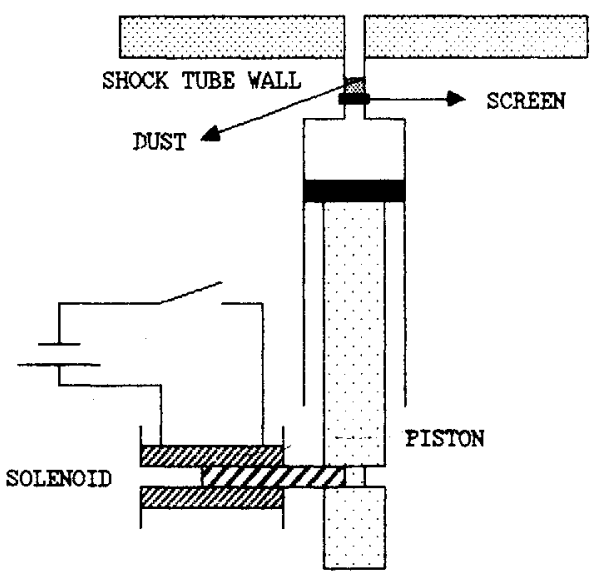

Fig. 1b Pneumatic dust injector.

Alignment Wires

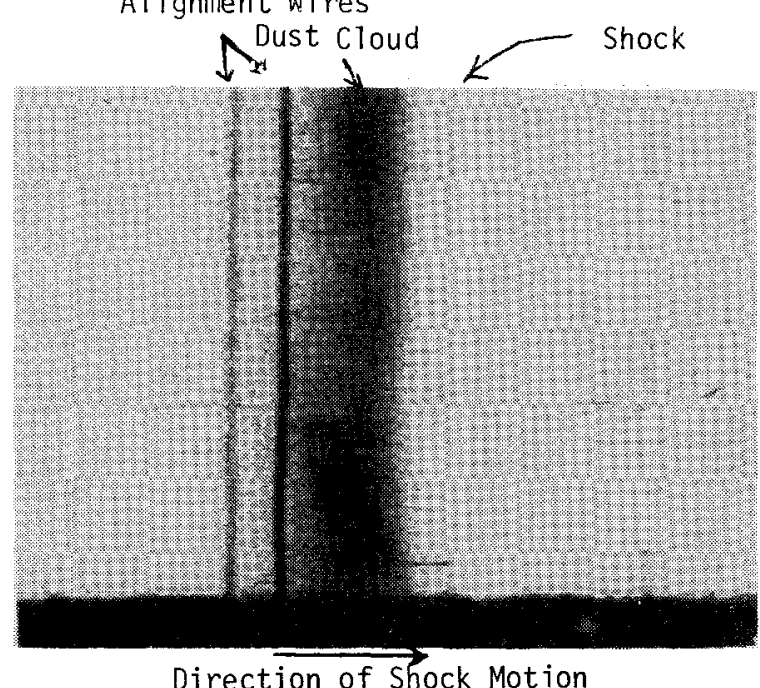

Direction of Shock Motion

Fig. 1c Shock wave passing a cloud of 53-74 $\mu \mathrm{m}$ graphite dust at $M=4.4$. Vertical alignment wires are $0.4 \mathrm{~mm}$ in diameter.

Table 1 Dust investigated

\begin{tabular}{lc}
\hline \hline Substance & $\begin{array}{c}\text { Particle size } \\
\text { range, } \mu \mathrm{m}\end{array}$ \\
\hline Coal (Pittsburgh Seam) & $53-74$ \\
Graphite & $53-74$ \\
Diamond & $4-6$ \\
Oats & $53-74$ \\
RDX-E & 2 (mean diameter) \\
RDX-E & 2 (mean diameter) \\
RDX-A & 37 (mean diameter) \\
\hline \hline
\end{tabular}

${ }^{\mathrm{a}}$ Implies addition of $10 \%$ cabosil by weight. 

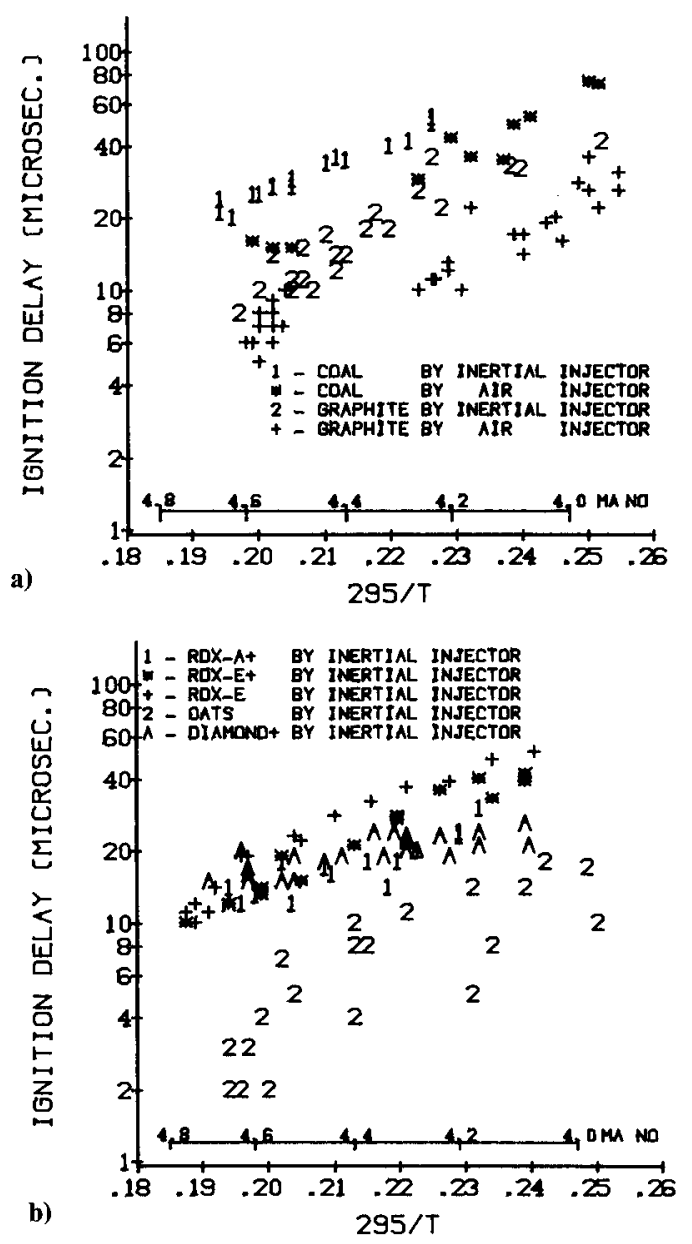

Fig. 2 Ignition delay data.

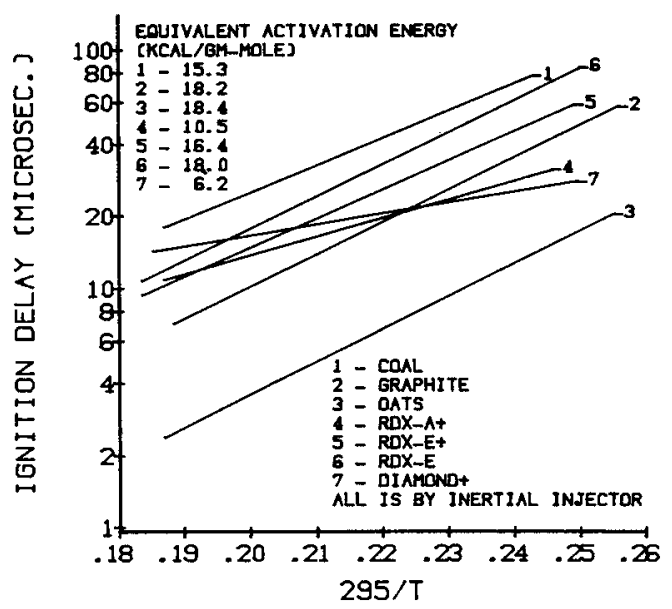

Fig. 3 Linear curves based on mean square fit to experimental ignition delay data.

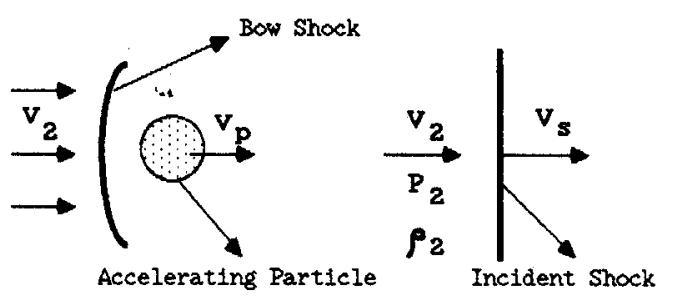

delay than RDX-E (mean diameter $2 \mu \mathrm{m}$ ) with cabosil. This supports the observed stronger detonability of RDX-A vs RDX-E, and may be related to the effect of the plastic coating on each particle. The ignition delay of the very small diamond particles is comparable to that of the much larger graphite particles, and is probably related to the surface properties of these two forms of carbon.

\section{Theoretical Analysis}

Immediately after shock passage each dust particle is in the supersonic flow induced by the shock wave. The resultant convective heating causes a rapid increase in the particle surface temperature. At the same time, the drag forces cause the particles to accelerate with an accompanying reduction in the relative velocity and the rate of convective heating. With increasing particle temperature the rate at which the gaseous oxidizer and the particle react increases until the reactive heat release results in temperature runaway or ignition. The rate of particle acceleration, which depends on the ratio of drag force to particle mass, will thus have an important influence on the ignition delay.

The theoretical model must account for particle acceleration, particle heating, and chemical reaction within the particle. In the present model, which follows that presented by Ural et al., ${ }^{2}$ it is assumed that the cloud is monodisperse with spherical particles, and that each particle is independent of the other particles in the cloud. The analysis then reduces to the consideration of a single accelerating spherical particle in a flow which is initially supersonic, as shown in Fig. 4.

The equation of motion of the particle is given by

$$
\frac{4}{3} \pi R^{3} \rho_{c} \frac{\mathrm{d} V_{p}}{\mathrm{~d} t}=\frac{1}{2} C_{D} \rho_{2} \pi R^{2}\left(V_{2}-V_{p}\right)^{2}
$$

The relative velocity $\left(V_{2}-V_{p}\right)$ ranges from supersonic to very low subsonic values, so that the drag coefficient $C_{D}$ varies widely; hence, it was necessary to use empirical correlations ${ }^{3}$ for $C_{D}$

Because of the high convective velocity around the particles, the Biot number, $B i=\left(h R / k_{c}\right)$, is of order unity, so that the temperature variation within the particle must be taken into account. Thus for $M=4.0,1.2<B i<2.6$, while for $M=4.8,2.2<B i<3.8$ for spherical, $53 \mu \mathrm{m}$ coal particles. The energy equation thus becomes the heat conduction equation for the particle interior:

$$
\frac{\partial T}{\partial t}=\frac{\alpha}{r^{2}} \frac{\partial}{\partial r}\left(r^{2} \frac{\partial T}{\partial r}\right)+\frac{u^{\prime \prime \prime}(r, t)}{\rho_{c} C}
$$

where $u^{\prime \prime \prime}$ is a source term that accounts for the heat released by the surface reaction within the porous particle. The boundary condition at the surface is

$$
k_{c} \frac{\partial T}{\partial r}(R, t)=h(t)\left[T_{f}(t)-T(R, t)\right]
$$

In formulating Eq. (3), it is assumed that the film conductance $h$ is uniform over the particle surface, even though this is actually not the case, so that the problem will remain spherically symmetric. Both the recovery temperature $T_{f}$ and $h$ will vary as the relative velocity $V_{2}-V_{p}$ decreases during particle acceleration. The empirical relations developed by Fox et al. ${ }^{4}$ have been used to determine $h$. Typical values of these parameters in the present case were:

for $M=4.0$ 
and for $M=4.8$

$$
2.0 \leq h \leq 3.1 \mathrm{cal} / \mathrm{cm}^{2} \text { s K } \quad 1900 \leq T_{f} \leq 2390 \mathrm{~K}
$$

It has been assumed that the ignition reaction is a firstorder surface reaction that, because of particle porosity, can occur on the pores within the particle. Hence, the source term $u^{\prime \prime \prime}$ has been taken as

$$
u^{\prime \prime \prime}=Q \rho_{c} S_{i} P_{\mathrm{O}_{2}} A \exp (-E / \bar{R} T)
$$

where $S_{i}$ is the internal surface area of the particle per unit mass, $Q$ the combustion heat release, and $A$ a preexponential factor. Evaluation of this source term is a key source of difficulty because of a lack of adequate kinetic data for many dusts of interest.

Equations (1-4) have been solved numerically for coal and oats dust. The thermal properties, internal surface area, and kinetic data for coal dust have been taken from Badzioch et al. ${ }^{5}$ Gan et al, ${ }^{6}$ and Field et al. ${ }^{7}$ The source of data for oats is given in Table 2, which summarizes the properties used in computing ignition delays of coal and oats dust. The internal surface area and activation energy of oats dust were chosen to provide the best fit between computed and measured ignition delay times. The solutions obtained display thermal runaway after a certain period of time has elapsed; this then is taken as the point of ignition. The results of these calculations are compared later to experimental data for $53 \mu \mathrm{m}$ coal and oats particles in Figs. 9 and 10 and agreement was found to be quite good.

As indicated above, because of a lack of sufficient data, the oats dust results are based on values of internal surface area and activation energy based on the best fit of theory and data. The theory, however, does provide a suitable framework for curve fitting between computed and measured data and may also provide a means of determining kinetic dust properties from ignition delay measurements.

\section{Characteristic Times}

From the preceding discussion it is evident that the ignition delay of dust particles is governed by at least three characteristic time scales: an acceleration time $\tau_{\mathrm{a}}$, a surface heating or thermal time $\tau_{\text {th }}$ and a chemical time $\tau_{\mathrm{ch}}$. As will be indicated below, a careful consideration of these time scales also leads to some approximate methods of computing ignition delay times.

From the equation of motion (1) of the particle it is readily shown that a characteristic time for acceleration is given by

$$
\tau_{\mathrm{a}}=\frac{R}{V_{2}} /\left[\frac{3}{8} \bar{C}_{D} \frac{\rho_{2}}{\rho_{c}}\right]
$$

where $\bar{C}_{D}$ is an average drag coefficient. As expected, the acceleration time increases with particle radius $R$ and with the ratio of the density of the particle material to that of the ambient gas $\left(\rho_{c} / \rho_{2}\right)$. Thus the small low density particles will rapidly accelerate to the gas velocity $V_{2}$ behind the shock. This behavior is strikingly illustrated in Fig. 5, which shows streak schlieren photographs of 18,88 , and $450 \mu \mathrm{m}$ aluminum oxide particles behind $M=3.64,3.77$, and 3.49 shock waves. The $18 \mu \mathrm{m}$ particles are almost instantly accelerated to the induced gas velocity behind the shock wave, while the ponderous $450 \mu \mathrm{m}$ particles are very slow to accelerate. Because of the importance of convection there is strong coupling between the acceleration time $\tau_{\mathrm{a}}$ and the rate of particle heating, and the rapid decrease of convection for small particles may account for the observation that clouds of fine particles are sometimes less detonable than clouds of larger coarser particles. The effect of radius on particle acceleration is also illustrated in Fig. 6, which shows the variation of relative Mach number with time for 53 and $74 \mu \mathrm{m}$ particles, computed using Eq. (1).

Chemical reactions will play an insignificant role during the initial stages of particle heating; hence, a solution of the unsteady heat conduction equation for a sphere with a convective boundary condition [Eq. (3)] can be used to determine the variations of surface temperature with time. Since this solution can be expressed in analytical form if a constant average value is used for the film conductance $h$, it also leads to a simple means of computing a characteristic thermal time $\tau_{\text {th }}$, i.e., the time required for the surface temperature $T_{s}$ to reach a given value. The solution given by Carslaw and Jaeger $^{9}$ is

$$
T=T_{f}+\frac{l}{r} \sum_{n=1}^{\infty} \beta_{n} \sin \left(\lambda_{n} r\right) \exp \left(-\alpha \lambda_{n}^{2} t\right)
$$

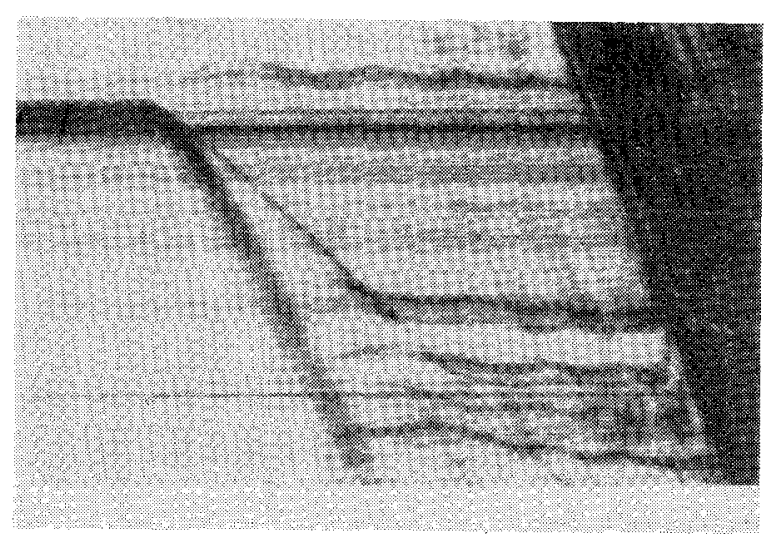

Fig. 5a Streak photograph of $18 \mu \mathrm{m}$ particles accelerated by an $M=3.64$ shock.

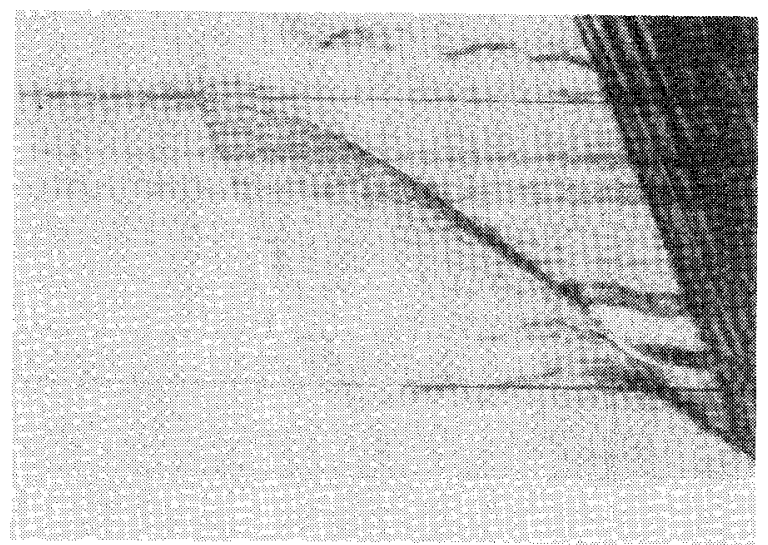

Fig. 5b Streak photograph of $88 \mu \mathrm{m}$ particles accelerated by an $M=3.77$ shock.

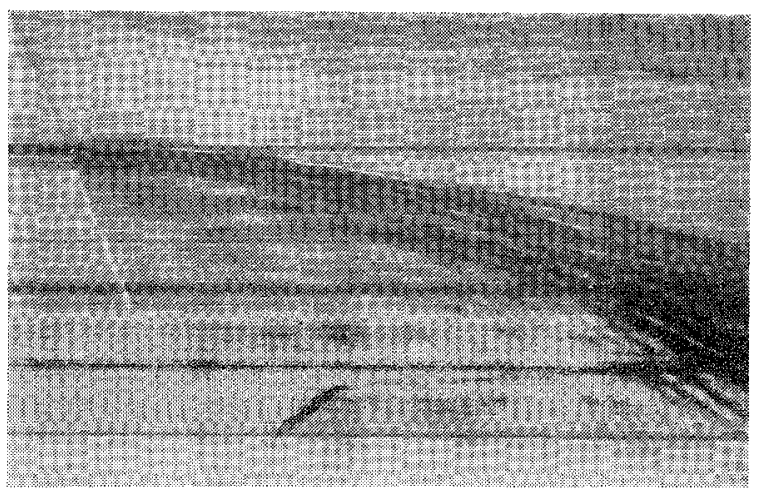

Fig. 5c Streak photograph of $450 \mu \mathrm{m}$ particles accelerated by an $M=3.49$ shock. 
Table 2 Thermal and chemical kinetic properties of coal and oats dusts

\begin{tabular}{lll}
\hline \hline & Coal & Oats \\
\hline Density, g/cm ${ }^{3}$ & 1.2 & $0.75^{\mathrm{a}}$ \\
$\begin{array}{l}\text { Thermal conductivity } \\
\text { cal } / \mathrm{c} \cdot \mathrm{s} \cdot{ }^{\circ} \mathrm{C}\end{array}$ & 0.00212 & $0.0007^{\mathrm{b}}$ \\
$\begin{array}{l}\text { Specific heat } \\
\text { cal } / \mathrm{m}^{\circ} \mathrm{C}\end{array}$ & 0.236 & $0.24^{\mathrm{b}}$ \\
$\begin{array}{l}\text { Heat of combustion, } \\
\text { cal } / \mathrm{g}\end{array}$ & 8559 & $3040^{\mathrm{c}}$ \\
$\begin{array}{l}\text { Internal surface } \\
\text { area } \mathrm{cm}{ }^{2} / \mathrm{g}\end{array}$ & $4.26 \times 10^{6}$ & $9 \times 10^{7^{\mathrm{d}}}$ \\
$\begin{array}{l}\text { Activation energy } \\
\mathrm{kcal} / \mathrm{g} \cdot \text { mole }\end{array}$ & 35.7 & $37.2^{\mathrm{d}}$ \\
$\begin{array}{l}\text { Pre-exponential } \\
\text { factor, } A \\
\left.\text { (g/cm }{ }^{2} \cdot \mathrm{s} \cdot \text { atm }\right)\end{array}$ & $8.71 \times 10^{3}$ & $3.1 \times 10^{4^{\mathrm{e}}}$ \\
\hline \hline
\end{tabular}

${ }^{a}$ Estimate based on bulk density of oats. ${ }^{b}$ Ref. 13, p. 122. ${ }^{c}$ Average value taken from Ref. 10. ${ }^{\mathrm{d}}$ Based on best fit of computed and measured ignition delays. ${ }^{e}$ Based on smoldering combustion measurements, Ref. 13.

where $\lambda_{n}$ are eigenvalues determined from

$$
\tan \left(\lambda_{n} R\right)=-\frac{\lambda_{n}}{A}
$$

with

$$
A=\frac{h}{k_{c}}-\frac{l}{R}
$$

The coefficients $\beta_{n}$ are given by

$$
\begin{aligned}
\beta_{n} & =\left\{4\left(T_{i}-\frac{B}{1+A R}\right)\left[\frac{\sin \left(\lambda_{n} R\right)}{\lambda_{n}}-R \cos \left(\lambda_{n} R\right)\right]\right\} \\
& \div\left[2 \lambda_{n} R-\sin \left(2 \lambda_{n} R\right)\right]
\end{aligned}
$$

where $T_{i}$ is the initial particle temperature and $B=$ $\left(h R / k_{c}\right) T_{f}$.

Because of the rapidly decreasing exponential term, two terms of the series in Eq. (6) are quite sufficient for determining $\tau_{\text {th }}$. Typical values of $\tau_{\text {th }}$ are shown for $53 \mu \mathrm{m}$ coal and oats dust in Figs. 7 and 8 with the logarithm of $\tau_{\text {th }}$ plotted vs the inverse surface temperature. Because of the different thermal properties of oats and coal the results are significantly different.

A characteristic chemical or reaction time $\tau_{\mathrm{ch}}$ can be defined on the basis of Eq. (4) as

$$
\tau_{\mathrm{ch}}=\exp (E / \vec{R} T) /\left(S_{i} P_{\mathrm{O}_{2}} A\right)
$$

For low values of the surface temperature $T$, the chemical time $\tau_{\text {ch }}$ will be much larger than the thermal time, as is evident from Figs. 7 and 8 , which show the variation of $\ln \tau_{\mathrm{ch}}$ with inverse temperature. As expected, these curves display the typical Arrhenius behavior.

With increasing surface temperature the thermal time $\tau_{\text {th }}$ increases rather slowly while there is a precipitous decrease in $\tau_{\mathrm{ch}}$ which is characteristic of high activation energy systems. This behavior suggests that ignition will occur soon after $\tau_{\mathrm{ch}}$ becomes less than $\tau_{\mathrm{th}}$, and one simplified approach to the determination of the ignition delay time is to choose the time at the point of intersection where $\tau_{\mathrm{th}}=\tau_{\mathrm{ch}}$. The results of this approach, which is equivalent to the introduction of an igni-

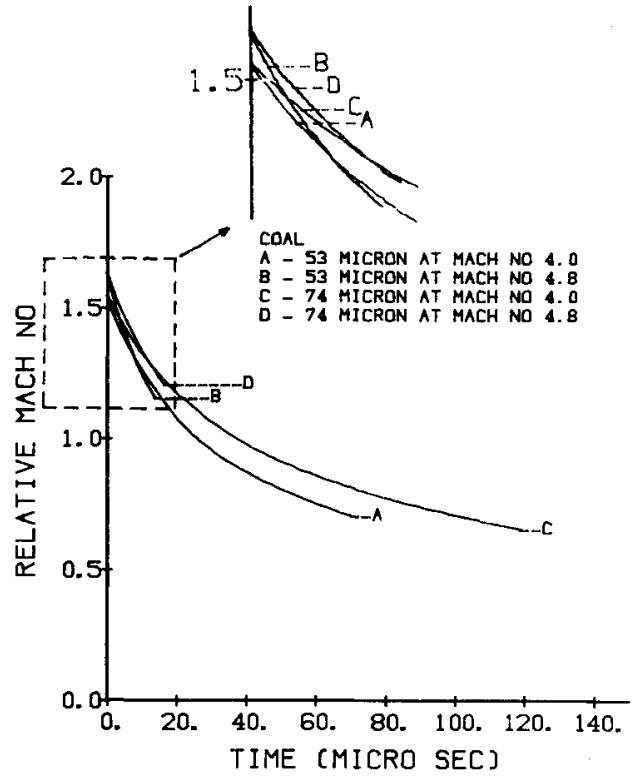

Fig. 6 Variation of relative Mach number with time for 53 and 74 $\mu \mathrm{m}$ dust particles.

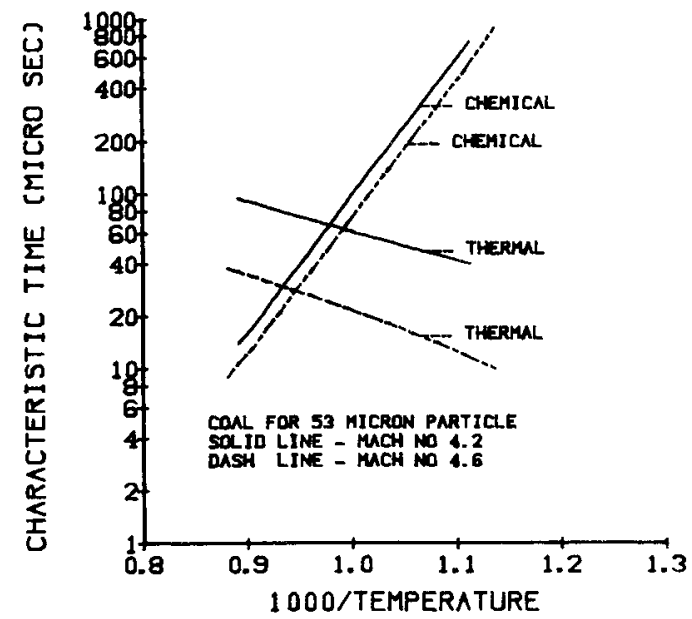

Fig. 7 Variation of characteristic thermal $\left(\tau_{\mathrm{th}}\right)$ and chemical $\left(\tau_{\mathrm{ch}}\right)$ time of coal particles with inverse surface temperature.

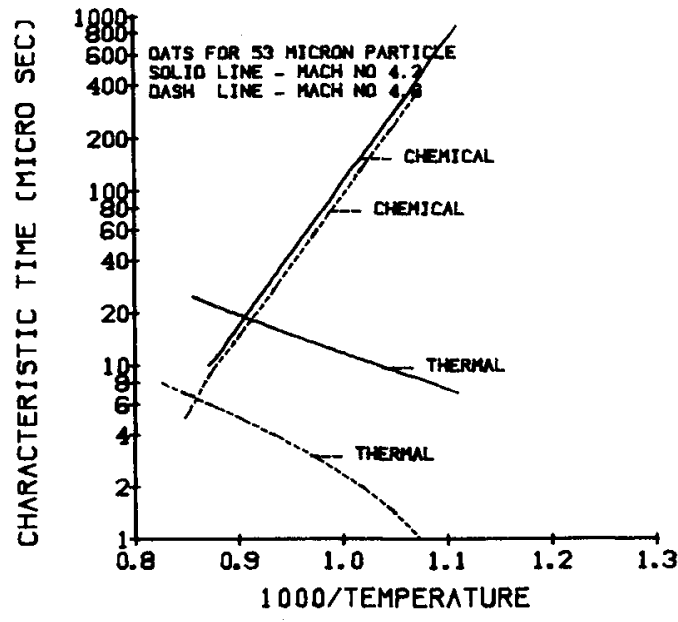

Fig. 8 Variation of characteristic thermal $\left(\tau_{\text {th }}\right)$ and chemical $\left(\tau_{\mathrm{ch}}\right)$ time of oats particles with inverse surface temperature. 


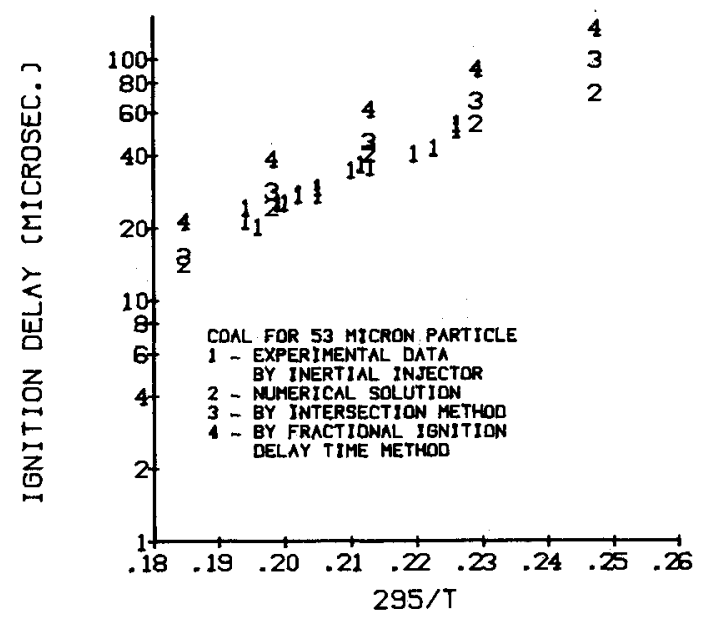

Fig. 9 Comparison of computed and measured ignition delays for coal dust.

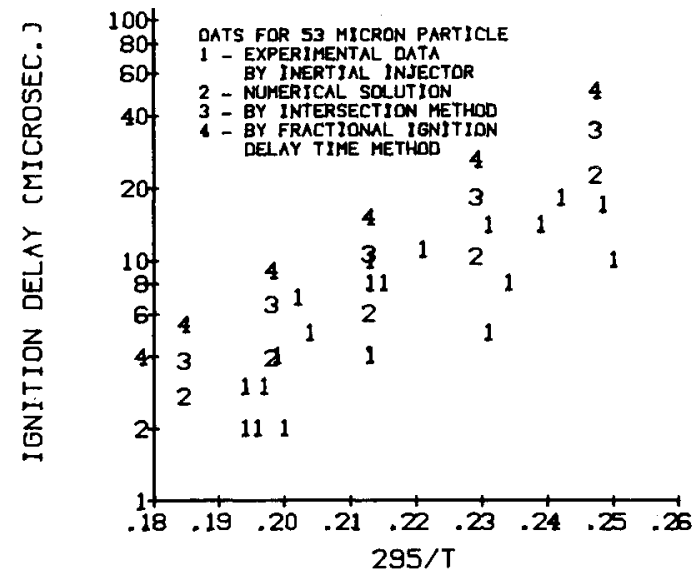

Fig. 10 Comparison of computed and measured ignition delays for oats dust.

tion temperature, are shown in Figs. 9 and 10 as the curves denoted by No. 3, and agreement with experimental results is quite good.

Another simplified approach to the calculation of the ignition delay time, which can be termed the fractional ignition delay time method, is based on the procedure employed by Gubin and Sichel ${ }^{11}$ and Oran et al., ${ }^{12}$ among others. The difficulty is, of course, that $\tau_{\mathrm{ch}}$ is constantly changing as the particle surface temperature changes during acceleration and convective heating. If the temperature were constant, $\tau_{\mathrm{ch}}$ would be of the order of the ignition delay time and during interval $\mathrm{d} t,\left(\mathrm{~d} t / \tau_{\mathrm{ch}}\right)$ represents the fraction of the elapsed ignition delay time. Thus

$$
\int_{0}^{\tau_{\mathrm{ig}}} \frac{\mathrm{d} t}{\tau_{\mathrm{ch}}}=1
$$

if $\tau_{\mathrm{ch}}=\tau_{\mathrm{ig}}$. This trivial result can be extended to the case in which $\tau_{\mathrm{ch}}(T)$ is a function of the temperature with $T=T(t)$ determined here by the rate of surface heating. Thus the ignition delay time is determined from the integral relation

$$
\int_{0}^{\tau_{\mathrm{ig}}} \frac{\mathrm{d} t}{\tau_{\mathrm{ch}}(T)}=1, \quad T=T(t)
$$

[from Eq: (6)]

The inverse of $\tau_{\mathrm{ch}}(T)$ is plotted vs time for coal and oats dust in Figs. 11 and 12, and because of the rapid increase of $\left(1 / \tau_{\mathrm{ch}}\right)$ after a given elapsed time, Eq. (9) will lead to a fairly

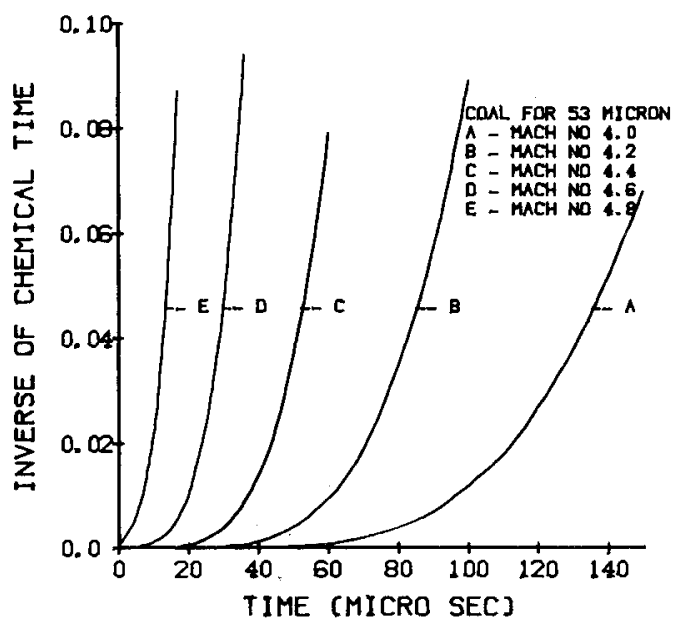

Fig. 11 Variation of inverse chemical time $\tau_{\mathrm{ch}}^{-1}$ with time for 53 $\mu \mathrm{m}$ coal particles.

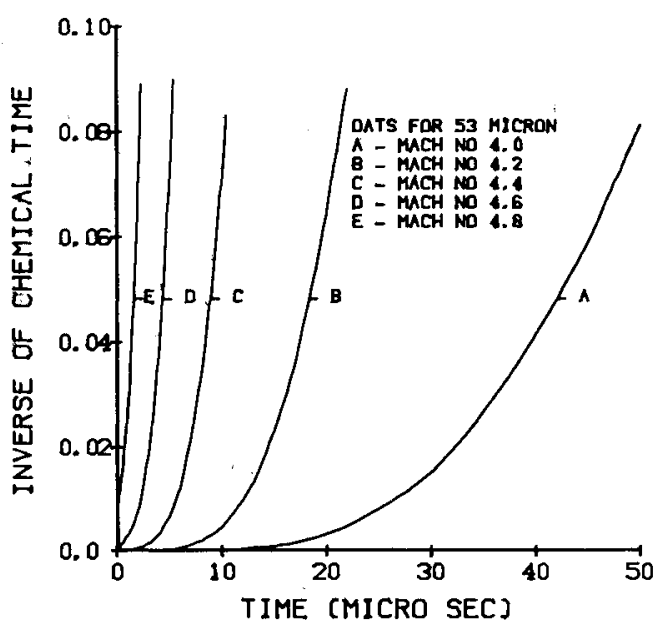

Fig. 12 Variation of inverse chemical time $\tau_{\mathrm{ch}}^{-1}$ with time for 53 $\mu \mathrm{m}$ oats particles.

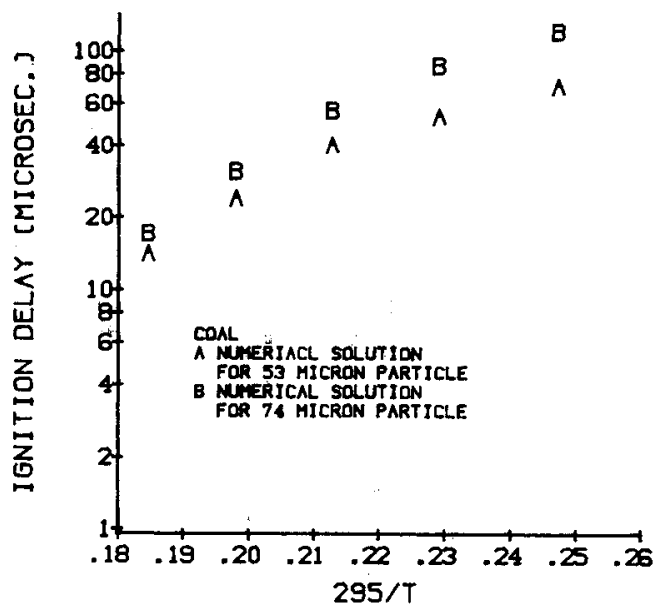

Fig. 13 Effect of particle diameter on ignition delay time.

sharp definition of $\tau_{\mathrm{ig}}$. This heuristic approach, which is closely related to the previous criterion that $\tau_{\text {ch }}=\tau_{\text {th }}$ at ignition, has no solid physical basis, but as shown in Figs. 9 and 10 is also in reasonable agreement with measurement.

\section{Discussion and Conclusions}

Ignition delay measurements have been made for a wide variety of dusts. Consistent insertion of a small controlled 
quantity of dust into the shock tube prior to shock arrival is one source of difficulty. Precise determination of the instant of ignition also poses a serious problem. Use of a photomultiplier tube to monitor radiation from the dust cloud, as was done here, does not provide an absolute measurement of particle surface temperature for an unequivocal indication of ignition. However, it is felt that the technique used here does provide reliable comparative measurements of the ignition delay of the various different types of dust considered in this investigation.

The theoretical model is based on the simultaneous solution of the equation of motion of the individual particles, and the heat conduction equation, including a reactive source term, for the temperature in the interior of the particle. These two equations are coupled by the dependence of the particle surface film conductance on the velocity of the particle relative to the surrounding gas. The dust cloud is assumed to consist of monodisperse spherical particles which do not interact with each other, while photomicrographs of dusts show irregular particles covering a considerable size range. The oats and coal dusts, which were the two dusts for which theory and experiment were compared, had particle diameters ranging from 53 to $74 \mu \mathrm{m}$.

The agreement between theory and experiment was remarkably good despite these many restrictive assumptions. The lack of kinetic data for most dusts was a major source of difficulty in computing ignition delay times using this theory described. While considerable data are available for coal, some of the key kinetic constants of oats dust were determined by comparing the computed and measured delay times. Thus, while the coal dust results support the main elements of the model, the theory only provides a reasonable framework for fitting computed and measured results by suitable choice of kinetic constants in the case of oats dust. These results also suggest that careful ignition delay measurements may provide a means of establishing the kinetic constants of a wide variety of dusts. It is interesting that the oats activation energy of $37.2 \mathrm{kcal} / \mathrm{mole}$ was very close to the value of $35.7 \mathrm{kcal} / \mathrm{mole}$ for coal dust, and is also of the same order as the activation energy of wheat dust in smoldering combustion. ${ }^{13}$

The calculated ignition delays reproduce the interesting experimental observation that the ignition process behaves as if it were governed by an Arrhenius reaction with an activation energy of $15-18 \mathrm{kcal} /$ mole. This occurs in spite of the fact that activation energies of the surface reactions which govern ignition are on the order of $35 \mathrm{kcal} / \mathrm{mole}$. This result suggests that ignition is governed by the physical process of particle heating rather than by surface reaction chemistry, a conclusion which is supported by the approximate analysis based on characteristic times.

These characteristic times have been identified: an acceleration time, a thermal time, and a chemical time. Convective heating is a key feature of particle ignition behind incident shocks, and its duration depends on the particle acceleration time. Thus, small particles rapidly accelerate to the local gas velocity, so that the time available for convective heating is short. This effect is, however, counterbalanced by the fact that the film conductance $h$ varies inversely with the particle radius. The chemical time is orders of magnitude greater than the thermal time during much of the particle heatup, suggesting that the dust ignition, at least in the cases analyzed here, is mainly a thermal phenomenon.
The approximate analysis based on this observation yielded results which were almost in exact agreement with the more detailed calculations.

For sufficiently short times the solution for the surface temperature of a sphere [Eq. (6)], which provides the basis for the approximate thermal analysis, is identical to that for a semi-infinite solid, suggesting that the particle diameter may only have a weak effect on ignition delay. This conclusion is supported by Fig. 13, which shows ignition delays computed for 53 and $74 \mu \mathrm{m}$ particles using the detailed theory.

While both the experiments and the theory involve many difficulties and approximations, the results should lead to a better understanding of particle ignition. A key conclusion is that incident shock ignition of particles is mainly a thermal process.

\section{Acknowledgments}

The authors would like to express their appreciation for support for the work reported here by the National Science Foundation under NSF Grant CPE-8023865, R. Rostenbach, Project Monitor, and by the Air Force Office of Scientific Research under AFOSR Grant No. 79-0093, B. J. Wolfson, Project Monitor.

\section{References}

${ }^{1}$ Kauffman, C. W., private communication, University of Michigan, Ann Arbor, MI, 1983.

${ }^{2}$ Ural, E. A., Sichel, M., and Kauffman, C. W., "Shock Wave Ignition of Pulverized Coal," Proceedings, 13th International Symposium on Shock Tubes and Waves, State Univ. of New York Press, 1982, pp. 809-817.

${ }^{3}$ Henderson, C. B., "Drag Coefficients of Spheres in Continuum and Rarefied Flows," AIAA Journal, Vol. 14, 1976, pp. 701-708.

${ }^{4}$ Fox, T. W., Rackett, C. W., and Nicholls, J. A., "Shock Wave Ignition of Magnesium Powders," Proceedings, 1lth International Symposium on Shock Tubes and Waves, Univ. of Washington, 1977, pp. 262-268.

${ }^{5}$ Badzioch, S., Gregory, D. R., and Field, M. A., "Thermal Conductivity and Thermal Diffusivity of Coal,"'Fuel, Vol. 43, 1964, pp. 267-280.

${ }^{6}$ Gan, H., Nandi, S. P., and Walker, P. L., "Nature of Porosity in American Coals," Fuel, Vol. 51, 1972, pp. 272-277.

${ }^{7}$ Field, M. A., Gill, D. W., Morgan, B. B., and Hawksley, P. G. W., "Combustion of Pulverized Coal," The British Coal Utilisation Research Association, Leatherhead, 1967.

${ }^{8}$ Kauffman, C. W., Nicholls, J. A., Sichel, M., and Lee, P., "Detonation Characteristics of Some Dusts and Liquid-Dust Suspensions," Draft Copy of Final Report to AFOSR, UM-016968-F, July 1983.

${ }^{9}$ Carslaw, H. S. and Jaeger, J. C., Conduction of Heat in Solids, Oxford, 1959.

${ }^{10}$ Martin, C. R., "Characterizationof Grain Dust Properties," ASAE Summer Meeting, Paper 78-3020, Logan, UT., 1978.

${ }^{11}$ Gubin, S. A. and Sichel, M., "Calculation of the Detonation Velocity of a Mixture of Liquid Fuel Droplets and a Gaseous Oxidizer," Combustion Science and Technology, Vol. 17, 1977, pp. 109-117.

${ }^{12}$ Oran, E. S., Boris, J. P., Young, T., Flanigan, T., Burks, T., and Picone, M., "Numerical Simulations of Detonations in Hydrogen-Air, and Methane-Air Mixtures," Eighteenth Symposium (International) on Combustion, The Combustion Institute, Pa., 1981.

${ }^{13}$ Leisch, S. O., "Smoldering Combustion in Horizontal Dust Layers," Ph.D. Thesis, Dept. of Aerospace Engineering, Univ. of Michigan, Ann Arbor, MI, Nov. 1983. 